\title{
Client Server Based Security Management System
}

\author{
Dr. May Paing Paing Zaw \\ Technological University (Thanlyin) \\ Yangon, Myanmar
}

\begin{abstract}
Nowadays, Network Management System (NMS) is a vital role and some key components of NMS include network device discovery, network device monitoring, network performance analysis and so many benefits. This paper aims to present a security management system for a own LAN based Client Server Network to control the security of a network using Java Programming Language. This system can provide the security feature for the user account setting and user management and proxy server feature. And all of the history of the security such as user account and proxy server history are kept in the java standard serializable file. So the user can view anytime the history of the security and proxy server.
\end{abstract}

Keywords: security management; TCP/IP based client server system; local area network

\section{INTRODUCTION}

A network management system is used to design, organize, analyze and administer computer and telecommunication networks, in order to maintain a desired level of service at all times. Network management software is designed to provide automated support for some or all of the network management functions. Network management software systems are used to perform some of the functions of monitors and analyzers, identify errors, run diagnostic tests, monitor entire an network, compile statistics, and prepare real-time management reports. The ISO network management model's five functional areas: such as configuration management, fault management, performance management, security management, accounting management and etc. Among them, security management is more important than others. Because it is very essential and useful in any fields[3,4].

\section{METHODOLOGY}

In general terms, LAN (Local Area Network) refers to a group of computers interconnected into a network so that they are able to communicate, exchange information and share resources (e.g. printers, application programs, database etc). A Local Area Network (LAN) is the result of connecting a number of computers or other IP devices together in a localized geographic area - for example in one room, building or several buildings. LANs are typically connected to each other via cable and more recently via radio waves. In an office building for example, workstations and personal computers (PCs) are commonly connected to each other with a Local Area Network. This allows an employees' equipment to communicate - send and receive files, share access to the files or data on another workstation even share applications [1].

TCP/IP (Transmission Control Protocol/Internet Protocol) is the basic communication language or protocol of the Internet. It can also be used as a communications protocol in a private network (either an intranet or an extranet). TCP/IP is a twolayer program. The higher layer, Transmission Control Protocol, manages the assembling of a message or file into smaller packets that are transmitted over the Internet and received by a TCP layer that reassembles the packets into the original message. The lower layer, Internet Protocol, handles the address part of each packet so that it gets to the right destination. Each gateway computer on the network checks this address to see where to forward the message. TCP/IP uses the client/server model of communication in which a computer user (a client) request and is provided a service (such as sending a Web page) by another computer (a server) in the network [2].

In this system, If a user logon to server, server checks client's username, password and decide which client should permit or not. Server identifies the user which connects the server and shows correspondence date in event viewer.

\section{CLIENT SERVER SYSTEM}

\subsection{Client/Server Paradigm}

The Client/Server paradigm has become a dominant one for the Internet. In this model, the clients are programs running on remote machines that communicate with a program called the server that runs at a single site and responds to requests from many clients. The server provides the clients with, say, Web pages or database information. Much of the World Wide Web is built on the client/server paradigm. The clients are Web browsers run by many millions of individual users, and the servers are the many Web hosting systems running at the many host sites on the Web [6].

A single server at a single host can support many hundreds or thousands or more of clients from around the world. Large systems that serve hundreds of thousands of clients balance the server load over multiple machines in an arrangement called "server farms." With Java you can build client/server systems with sockets or with RMI (Remote Method Invocation). In a socket based client/server system, a server listens to a particular port for client applications sending requests for connections. A Server Socket class is provided in Java that allows for a server to monitor and answer such requests for connections. The client sends the request for a connection by creating a socket with the host name and port for that server as discussed in the previous section. 


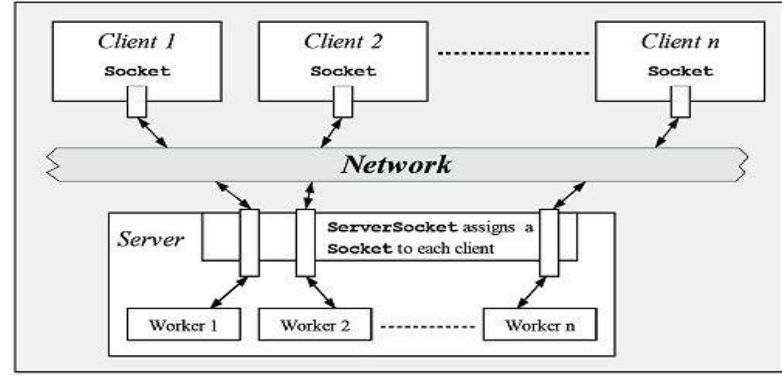

Figure 1 The basic aspects of a socket based client/server system

The above figure shows a diagram illustrating the basics of a socket-based client/server system. The Server Socket instance listens for a client to connect to the particular port. When a client request arrives, the Server Socket object sets up a Socket instance for the connection and then spins off a new thread to interact with the client via that socket. Many clients can therefore be served since each client has an independent thread dedicated to it [5].

\subsubsection{Class Server Socket (TCP Server Connections)}

A ServerSocket is a mechanism by which a server can accept connections from clients across a network. The server will open the ServerSocket and wait or listen for connections from clients. The ServerSocket class creates a Socket for each client connection. The server then handles the connections in the normal manner with Input and Output streams.

\subsubsection{Class Client Socket (TCP Client Connections)}

A Socket is a Java representation of a TCP network connection. In order to communicate with a remote host the Java client must first create a Socket, which will establish the TCP connection. In doing so a host name and port number must be specified. There must be a server actively listening on the specified port or the connection will fail with IOException. These constructors allow the Socket connection to be established.

\subsection{Security Management System Design}

In this system, the system will check all of the user which will connect from the clients and if this user is valid user of the system, the server will allow to enter the server and if invalid user will connect the system can not accept and resend to the client that the user is invalid and all of these messages are kept in the database file. Moreover in this system, there is the user management function to disable or enable the user account. If the disable user will connect from client to server, this system will send the message to the client that the client is connecting the server with the disable user. And this message will save to the data file with the detail time. So the system can view the message of the security that which client is connected to the server with which user at when. And moreover, this system can provide the proxy server feature. So this system can save the track of the proxy server message in the data file.

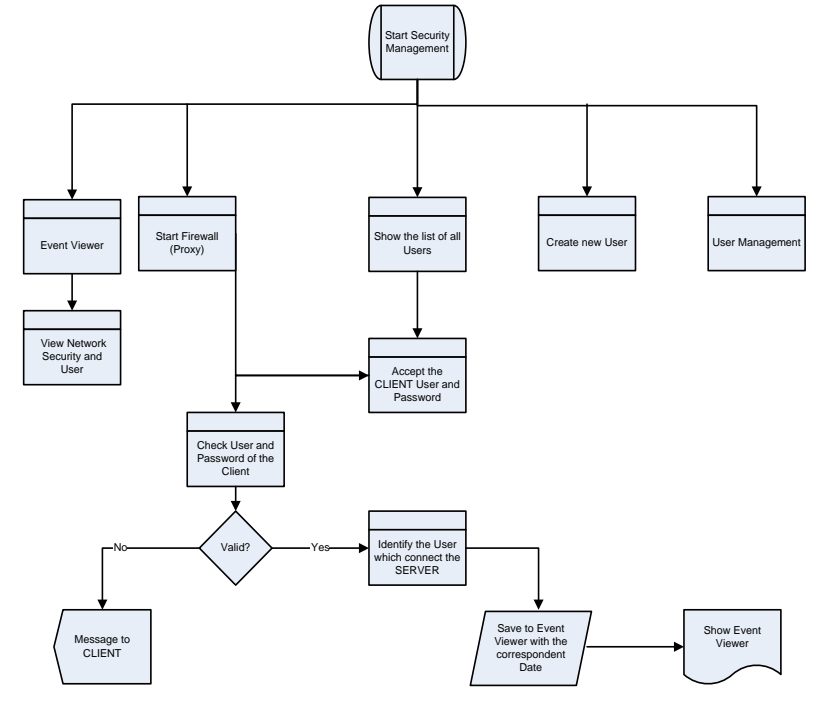

Figure 2.User Account Entry

\section{TEST AND RESULTS OF THE SYSTEM}

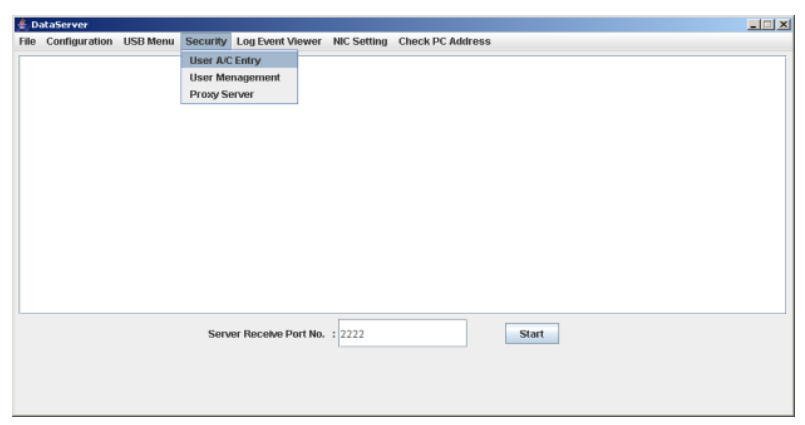

Figure 3.Security Menu

There are three sub menu of the security feature such as user Account Entry and user Management and proxy Server menu. In this menu, new users are added by this program and for the new user, user id and password and confirm password must be provided. And Password and Confirm password also must be the same in detail. And if the OK button is pressed, all of the user information will save in the Java Serializable object file. This file is to save all of the information of the user in the form of Ascii character. If the cancel button is pressed the user entry screen will clear and exit the program. 


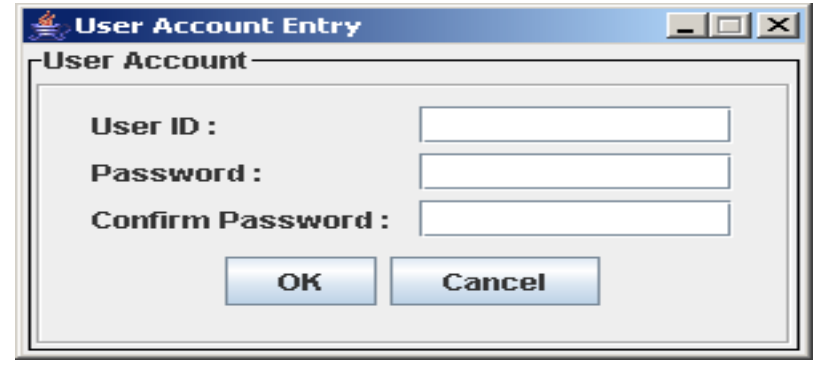

Figure 4.User Account Entry

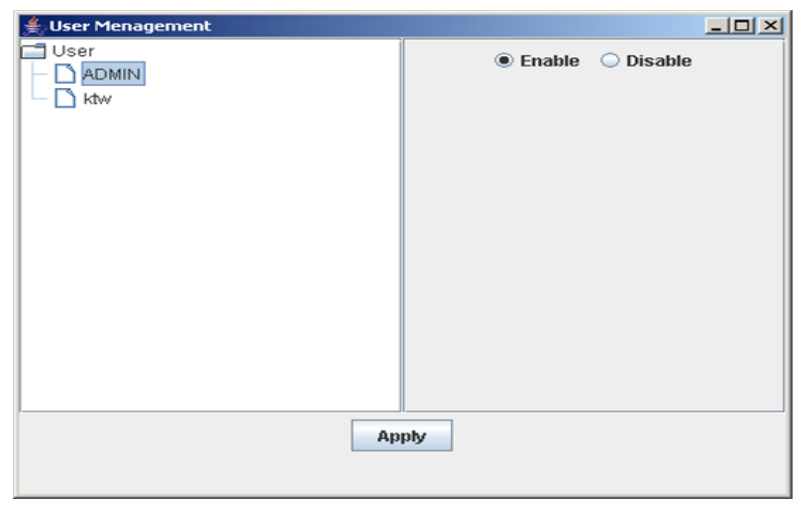

Figure 5. User Account Management Entry

User enable and disable feature can be updated by this program. When the user name node is pressed the user state is showed at the right side of the screen and the radio button will show the user is enable or disable. And when the user want to change the properties of the user id, click the radio button and then pressed the apply button. Then the user properties will save in the user profile file. This file is also java Serializable file.

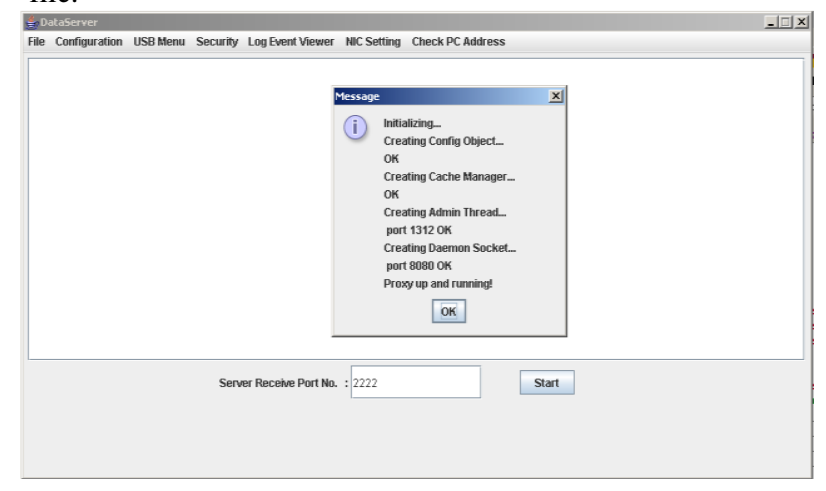

Figure 6. Proxy Server Menu

When the proxy server is on from the security sub menu, the proxy server will work. if the proxy server is actually work the message will show and Proxy up and running message will appear.

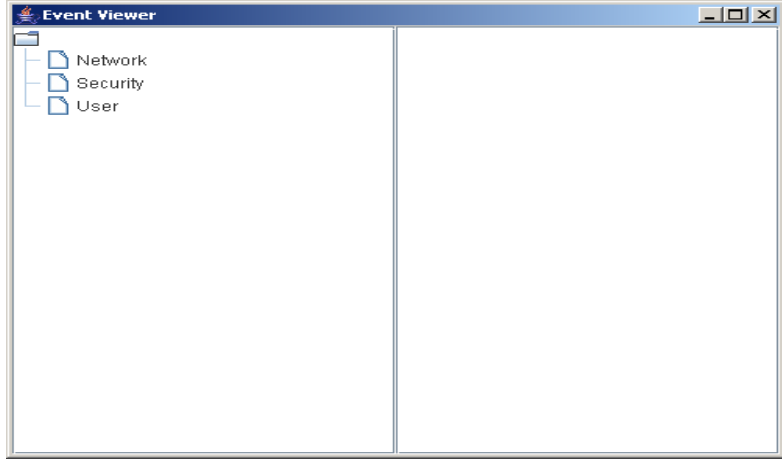

Figure 7. Log Event Viewer Menu

There are three type of the log event viewer such as Network, Security and User. When the network node of the Event Viewer program is clicked, all of the network history event which are concerned of the client program and which user is connected form which client at which time and which invalid user is connected at which time to server will show at the right side of the program. So the user can view the all of the network history of the client and server connected history and $\log$ in user history as shown in figure 7.

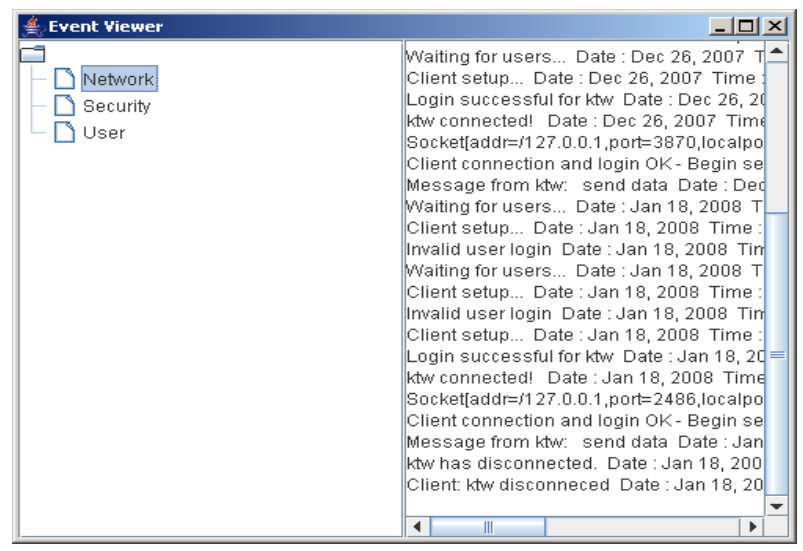

Figure 8. Network Event Viewer

\begin{tabular}{|c|c|}
\hline Event Viewer & $-|a| x \mid$ \\
\hline $\begin{array}{l}\square \text { Network } \\
\square \text { Security } \\
\square \text { User }\end{array}$ & 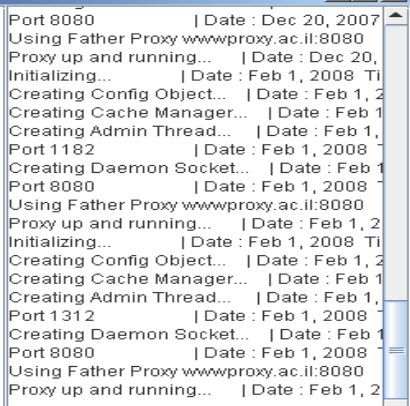 \\
\hline & \begin{tabular}{||l|l|l|}
4 & ||$l \mid$ & \\
\end{tabular} \\
\hline
\end{tabular}

Figure 9. Network Security

When security node is pressed, the history of the network security history will show. In this message history, there are so many messages about the proxy server start up time and the message of the port using the server program. 


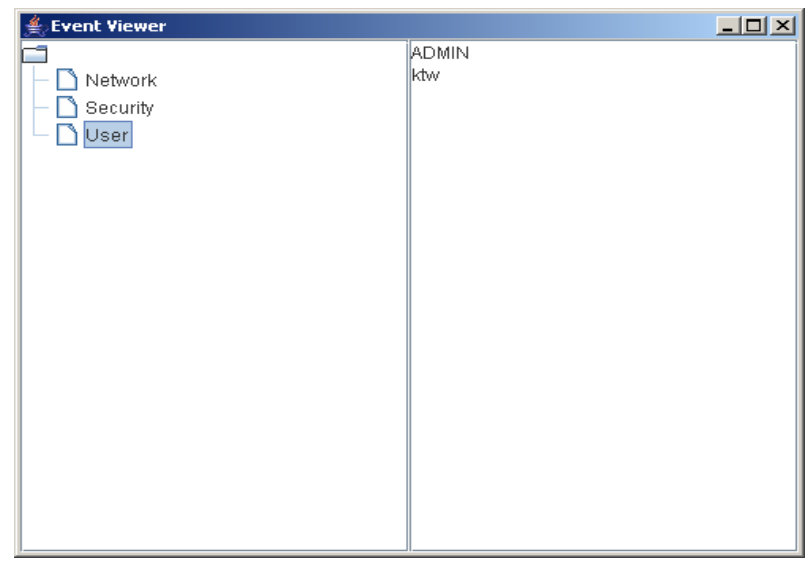

Figure 10. Users Control Menu

When user node is pressed, list all of the user who are create and accepted in the server program.

\section{ACKNOWLEDGMENTS}

Special thanks to my rector, Dr. Theingi from Technological University (Thanlyin), for her kindly support, encourage and valuable advice.

\section{Conclusion}

In this client server based network security system, the server can listen all of the requests of the clients and response to the clients. This system can provide the security feature for the user account setting and user management and proxy server feature and all of the history of the security such as user account and proxy server history are kept in the java standard serializable file. This system is used java programming language so all of the programs in the system are developed by java language and native dll file. But in this system, It is not implemented the function of which user has to invoke which service of the server and user level. So in the future, the user level function should include.

\section{REFERENCES}

[1] www.wikipedia.com, Local Area Network.

[2] Craig Hunt, 2002. TCP/IP Network Administration TCP/IP Guide, Third Edition.

[3] encyclopedia. thefreedictionary.com, Network

Management System

[4] Mr. Musa Odero, The Role of Network Management in the Emerging Infrastructure.

[5] Core Java (Students Guide), Java 2 Platform (StandardEdition) and the Java Developers Almanac

$<$ http://www.javaabout.com/ad>and $<$ http://www.sun.com>

[6] Computer Network Study Guide with Microsoft NT 4.0 